\title{
Two-Photon Cavity Solitons in Active Optical Media
}

\author{
R. Vilaseca, M. C. Torrent, and J. García-Ojalvo \\ Departament de Física i Enginyeria Nuclear, Universitat Politècnica de Catalunya, Colom 11, E-08222 Terrassa, Spain
}

M. Brambilla

Dipartimento di Fisica and INFM, Politecnico di Bari, Via E. Orabona 4, I-70126 Bari, Italy

\section{San Miguel}

Instituto Mediterráneo de Estudios Avanzados, Universitat de les Illes Balears, E-07071 Palma de Mallorca, Spain

(Received 29 September 2000; published 3 August 2001)

\begin{abstract}
We show that broad-area cascade lasers with no absorbing intracavity elements support the spontaneous formation of two-dimensional bright localized structures in a dark background. These cavity solitons consist of islands of two-photon emission embedded in a background of single-photon emission. We discuss the mechanisms through which these structures are formed and interact, along with their properties and stability.
\end{abstract}

DOI: $10.1103 /$ PhysRevLett.87.083902

PACS numbers: 42.65.Sf, 42.50.Hz, 42.65.Tg

Spatial solitons are among the most fascinating nonlinear localized structures exhibited by spatially extended systems. In an optical context, these localized structures take the form of self-trapped light beams that propagate through nonlinear media with an unperturbed transverse cross section, and result from a dynamic balance between a spreading linear mechanism (diffraction) and a nonlinear shrinking one [1]. Spatial solitons might be useful for all-optical parallel signal transmission and processing.

Although spatial solitons were initially associated with conservative systems, investigations in past years have shown that similar structures can also appear in dissipative systems. Presently there is a large interest in investigating the case of a nonlinear optical medium placed within a cavity (or near a mirror), in which the influence of feedback and resonance effects leads to localized structures with distinctive qualitative and quantitative characteristics [2]. These cavity solitons (CS) often result from a bistability between two stationary spatially extended states of the system, in the form of either self-trapped switching waves between two homogeneous states, or isolated pattern elements embedded in a homogeneous background. They have been predicted or observed thus far in passive (i.e., externally driven) media (with absorptive/dispersive [3-5], photorefractive [6], second-order [7], and thirdorder [8] nonlinearities), and also in lasers with a saturable absorber [9].

Extensive studies of the spatiotemporal dynamics of two-level lasers (see, e.g., [10]) suggest that CSs do not exist in purely active single-photon lasers (i.e., without absorbing intracavity elements). In this paper, we show that CSs can in fact exist in purely active media, provided that single-photon amplification is complemented with two-photon emission processes. As we show below, the presence of these fundamental light-matter interaction processes leads to the formation of CSs with distinctive features, such as the spatial separation of one- and two-photon amplification, their moderately long interaction range, and their globally attracting character (they are the only stable emission regime of the system).

We consider a cascade laser, in which two fields of different frequency are simultaneously generated through their coupling with the two consecutive transitions defined by a three-level atomic system (Fig. 1). Interaction between these two fields comes through the population of the intermediate level 0 and the two-photon quantum coherence between levels 1 and 2. Transverse effects have been seen to lead in this system to coupled patterns of the two generated fields, in both low-Fresnel-number [11] and high-Fresnel-number (broad-area) [12] cavities. In what follows, we will concentrate on the latter case. The spatiotemporal behavior of such a cascade laser can be described by the Maxwell-Bloch equations [12]:

$$
\begin{aligned}
\dot{\alpha}_{1}= & -\sigma_{1} \alpha_{1}+i \sigma_{1} \rho_{01}+i a_{1} \nabla^{2} \alpha_{1} \\
\dot{\alpha}_{2}= & -\sigma_{2} \alpha_{2}+i \sigma_{2} \rho_{20}+i a_{2} \nabla^{2} \alpha_{2} \\
\dot{\rho}_{01}= & -\left(1+i \delta_{1}\right) \rho_{01}-i \alpha_{1} d_{1}+i \sqrt{\chi} \alpha_{2}^{*} \rho_{21} \\
& +\beta_{1} \xi_{1} \\
\dot{\rho}_{20}= & -\left(1+i \delta_{2}\right) \rho_{20}-i \alpha_{2} d_{2}-i \alpha_{1}^{*} \rho_{21} / \sqrt{\chi} \\
& +\beta_{2} \xi_{2} \\
\dot{\rho}_{21}= & -(\Gamma+i \epsilon) \rho_{21}-i \sqrt{\chi} \alpha_{1} \rho_{20}+i \alpha_{2} \rho_{01} / \sqrt{\chi} \\
\dot{d}_{1}= & -b d_{1}-2 \operatorname{Im}\left(\alpha_{1} \rho_{01}^{*}\right)+\chi \operatorname{Im}\left(\alpha_{2} \rho_{20}^{*}\right) \\
\dot{d}_{2}= & b\left(r_{2}-d_{2}\right)+\chi^{-1} \operatorname{Im}\left(\alpha_{1} \rho_{01}^{*}\right)-2 \operatorname{Im}\left(\alpha_{2} \rho_{20}^{*}\right)
\end{aligned}
$$

where $\alpha_{1}$ and $\alpha_{2}$ are the complex envelopes of the generated electric fields, $\rho_{i j}$ the complex one- and twophoton coherences, $d_{1}$ and $d_{2}$ the population inversions of the two transitions, $r_{2}$ the pumping rate for the upper level 2, $a_{1}$ and $a_{2}$ the diffraction coefficients, $\sigma_{i}, \Gamma$, and $b$ the relaxation rates, and $\chi$ the efficiency of transition 1 versus transition 2 . The cavity detunings for the two fields 


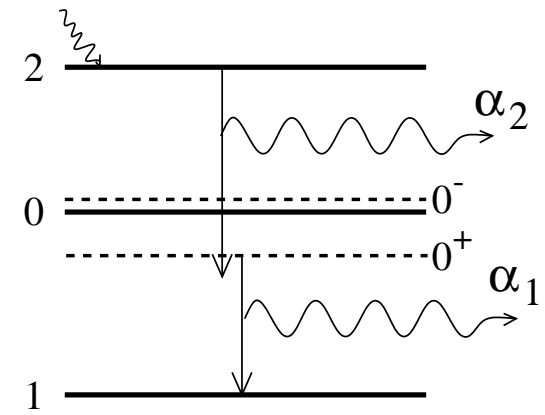

FIG. 1. Energy-level scheme of a cascade laser. Arrows indicate the cavity frequencies.

are given by $\delta_{i} \equiv \omega_{\mathrm{tr}, \mathrm{i}}-\omega_{\mathrm{cav}, \mathrm{i}}$ (with $\omega_{\mathrm{tr}, \mathrm{i}}$ denoting the transition frequency and $\omega_{\text {cav,i }}$ the cavity resonance frequency), and $\epsilon=\delta_{1}+\delta_{2}$. Spontaneous emission processes are accounted for by Gaussian white noise terms $\xi_{i}(\vec{x}, t)$ with zero mean and intensity $\beta_{i}^{2}$.

Since pumping is assumed to act only on the upper transition, laser emission occurs first, for increasing pumping rate, only in field $\alpha_{2}$. If the cavity detuning for this field is negative $\left(\delta_{2}<0\right)$, which is the situation that we will assume in what follows, then it is well known [10] that the transverse profile of the field is homogeneous and its frequency $\omega_{L}$ is given by the pulling formula $\delta_{L}=\delta_{2} /\left(\sigma_{2}+1\right)$, where $\delta_{L}=\omega_{\mathrm{tr}, 2}-\omega_{L}$. Field $\alpha_{2}$ induces an ac-Stark splitting of level 0 (Fig. 1), so that field $\alpha_{1}$, when generated, has two possible resonance frequencies (Autler-Townes doublet) occurring at cavity detunings given by $\delta_{1}^{ \pm}=\frac{1}{2}\left(-\delta_{L} \pm \sqrt{\delta_{L}^{2}+4 \alpha_{2}^{2}}\right)$. These values correspond to resonance with levels $0^{+}$and $0^{-}$, and are associated, for large $\left|\delta_{2}\right|$, to two-photon and step-by-step (i.e., single-photon) processes, respectively [13].

Let us focus on the case of two-photon resonance, $\delta_{1}=$ $\delta_{1}^{+}$. In the absence of transverse effects $\left[a_{1}=a_{2}=0\right.$ in Eqs. (1), homogeneous-field approximation], Fig. 2 shows (in continuous and dashed lines) the steady-state intensities versus the pump parameter $r_{2}$, for $\left|\delta_{2}\right|$ large $\left(\delta_{2}=-20\right)$ and $\delta_{1}=\delta_{1}^{+}$. By increasing $r_{2}$, the zero-intensity solution first undergoes a supercritical pitchfork bifurcation (PB) at $r_{2}=r_{2}^{\mathrm{PB}}$, leading to emission of field $\alpha_{2}$, followed by a subcritical PB at $r_{2}=r_{2}^{\text {th }}$, which leads to emission of $\alpha_{1}$ as well. Because of this second bifurcation, the system abruptly jumps to a two-photon branch with large intensity for both fields $\alpha_{1}$ and $\alpha_{2}$, because of the weak initial saturation of two-photon processes [14]. As shown in Fig. 2, two domains of bistability appear: one for $r_{2}^{\mathrm{SN}}<$ $r_{2} \leq r_{2}^{\mathrm{PB}}$, involving the two-photon (upper) branch and the zero-intensity solution, and another for $r_{2}^{\mathrm{PB}}<r_{2} \leq$ $r_{2}^{\text {th }}$, involving the two-photon branch and the single-field branch. As will be shown below, the second of these domains will be at the origin of CS formation.

When transverse effects are taken into account, important changes take place. First, both resonances of the field $\alpha_{1}$ with the atomic levels $0^{-}$and $0^{+}$can occur simultane-

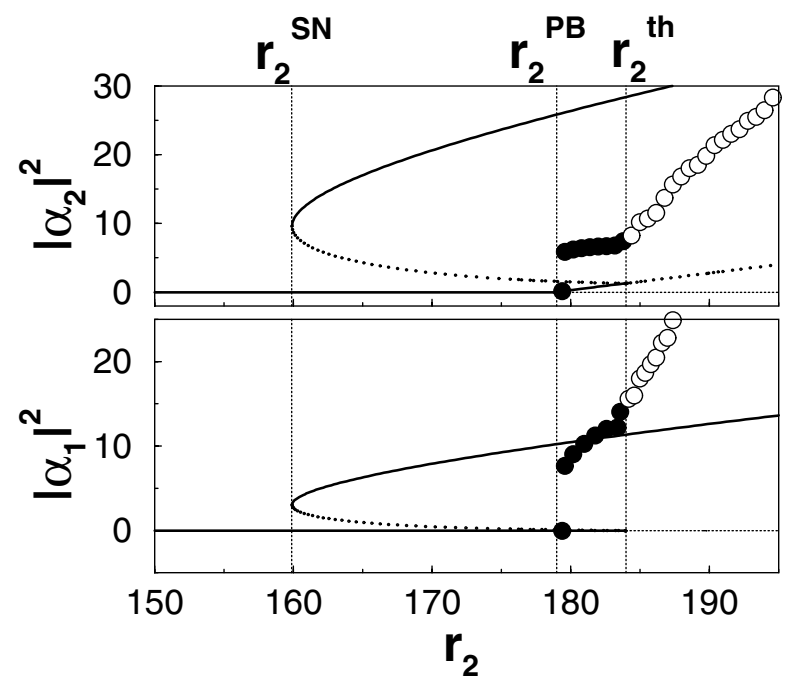

FIG. 2. Field intensity vs pumping for a cascade laser, for $\delta_{2}=-20$ and $\delta_{1}=13.33 \approx \delta_{1}^{+}$. Solid (dashed) lines: stable (unstable) steady solutions in the homogeneous-field limit. Full circles: soliton peak intensity; empty circles: time-averaged peak intensity $\left(\sigma_{1}=\sigma_{2}=0.5, b=\Gamma=1\right.$, and $\left.\chi=0.76\right)$.

ously, for the same value of the cavity detuning $\delta_{1}$ [13]. This is shown in Fig. 3(a), which represents the neutral stability curve of the state $\left(\alpha_{1}=0, \alpha_{2} \neq 0\right)$ for the same parameter values of Fig. 2. In Fig. 3(a), $k$ represents the wave number of a traveling-wave perturbation of $\alpha_{1}$. Above the neutral stability curve, the perturbation grows. Minima occur at two values of $|k|$, given by $|k|=0$ and $|k|=\sqrt{\delta_{1}^{+} / a_{1}}$. The first value means generation of a homogeneous field $\alpha_{1}$ due to resonance with level $0^{+}$(two-photon emission). In contrast, the second value means generation of a transverse traveling wave for $\alpha_{1}$, to be in resonance with level $0^{-}$(step-by-step emission). As in two-level lasers [10], generation of the transverse component leads to emission of a tilted wave with an increased wave number and frequency.

As shown in Fig. 3(a), the threshold is exactly the same for the two minima (dashed line), and coincides with that found above in the case of the homogeneous-field approximation $\left(r_{2}^{\text {th }}\right)$. Just below this threshold, the spontaneous
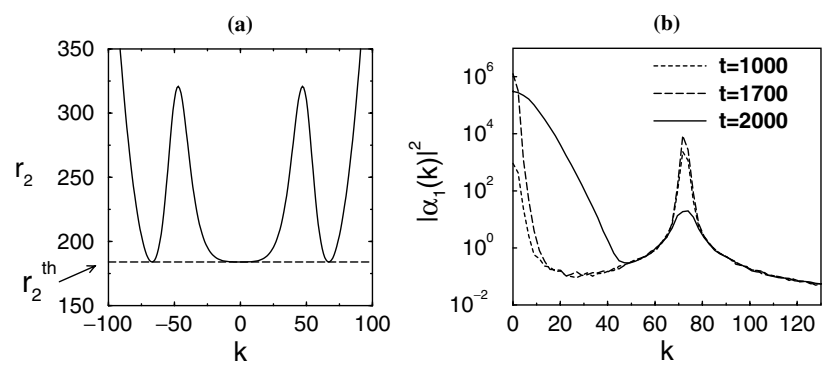

FIG. 3. (a) Neutral stability curve of $\left(\alpha_{1}=0, \alpha_{2} \neq 0\right)$ for $\delta_{2}=-20.0$ and $\delta_{1}=13.33$. (b) Spherically averaged far-field intensity of $\alpha_{1}$ for $r_{2}=184$, at three instants of time. Other parameters are those of Fig. 2, plus $a_{1}=a_{2}=0.003$. 
(noise-induced) emission on field $\alpha_{1}$ occurs predominantly through single-photon, step-by-step processes (i.e., with $|k|=\sqrt{\delta_{1}^{+} / a_{1}}$ ). However, when pumping is increased above $r_{2}^{\text {th }}$, the smaller initial saturation of two-photon processes makes them take over the nonlinear competition between the two wave numbers. This can be seen in Fig. 3(b), which portrays the spherically averaged far-field intensity of field $\alpha_{1}$ at three instants of time, showing how the two-photon wave number $(|k|=0)$ eventually becomes larger (and broader) than its single-photon counterpart $\left(|k|=\sqrt{\delta_{1}^{+} / a_{1}}\right)$. The result is that, after a short transient, emission of field $\alpha_{1}$ occurs in the form of cavity solitons, as shown in Fig. 4. This figure displays typical intensity and phase profiles of the two fields [see also Figs. 5(a) and 5(b)]. These CS show at least three remarkable distinctive features.

(i) They appear spontaneously as pumping is increased above its threshold value, independently of the initial state of the system. In other words, they are the only stable emission state of the system: All homogeneous and travelingwave solutions are unstable. The CS density is a growing function of the pump power. This fact entails a response to addressing actions (e.g., via an external pulse) very different from other classes of CSs: Writing (erasing) a new CS leads here to the disappearance (appearance) of another CS in a nearby position. This could lead to a different class of all-optical logical operations. (a)

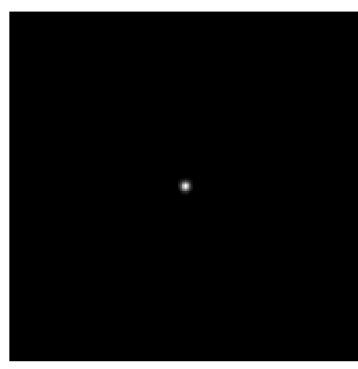

(c)

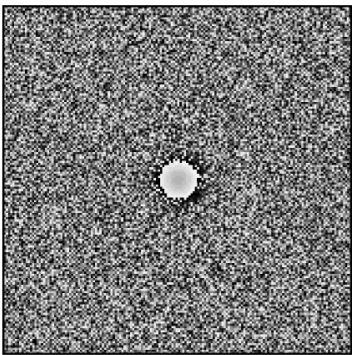

(b)

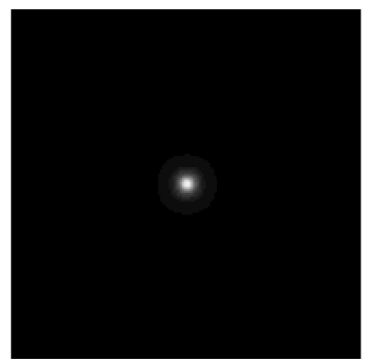

(d)

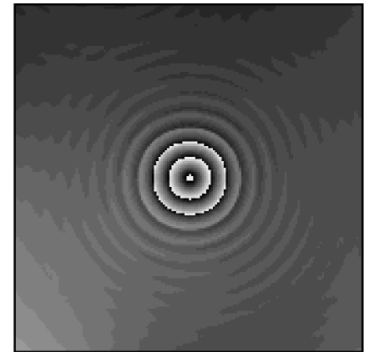

FIG. 4. Transverse emission of a broad-area cascade laser for the parameters of Fig. 3(a), plus $r_{2}=179.4$ and $\beta_{1}=\beta_{2}=$ $10^{-5}$ : intensity [(a) and (b)] and phase [(c) and (d)] profiles of fields $\alpha_{1}$ [(a) and (c)] and $\alpha_{2}$ [(b) and (d)]. In all plots, maxima is represented by white, and minima by black. In the phase plots, the scale ranges from $-\pi$ to $+\pi$. (ii) The CS formation brings about a spatial separation between single-photon and two-photon processes, as shown by the two-photon coherence profile of Fig. 5(c): The two-photon processes accumulate in the CS, whereas all the background is formed by single-photon processes. Because of that, the soliton exhibits no population inversion between levels 0 and 1 [Fig. 5(d)], and the noisy background of field $\alpha_{1}$ [Fig. 4(c)] exhibits the dominant transverse wave number corresponding to the outer minimum of Fig. 3(a). These facts may indicate that CS formation is related to the homogeneous-field bistability domain $r_{2}^{\mathrm{PB}}<r_{2}<r_{2}^{\text {th }}$ (Fig. 2). However, we should note again that none of these homogeneous solutions is stable in the presence of transverse diffraction.

(iii) The spatial profiles of the two fields $\alpha_{1}$ and $\alpha_{2}$ are different: Whereas field $\alpha_{1}$ displays a strongly localized structure similar to that of bright solitons in many other optical systems, field $\alpha_{2}$ extends up to longer distances, taking the form of a target pattern that emits damped phase waves coupled to damped intensity waves. This fact gives rise to a combined short/long interaction range which contrasts with the properties of standard CSs (e.g., the maximum soliton packing density is here more limited than usual), and leads to interesting dynamics: The structures are well shaped and localized in field $\alpha_{1}$, but at the same time they can interact (through field $\alpha_{2}$ ) with other moderately distant CSs. The origin of this different spatial profile of the two fields is not yet clear. In any case, there is not any supercritical Hopf bifurcation [15] of the lower stability branch in Fig. 2 that could give rise to the concentric rings of field $\alpha_{2}$.

For increasing pumping, as the CS density increases, the solitons start interacting through the rings of field $\alpha_{2}$ mentioned above, which leads to progressively complex dynamics, eventually producing turbulent motion and birth/death of solitons. Empty circles in Fig. 2 correspond to this regime (full circles denote stable CS), and represent

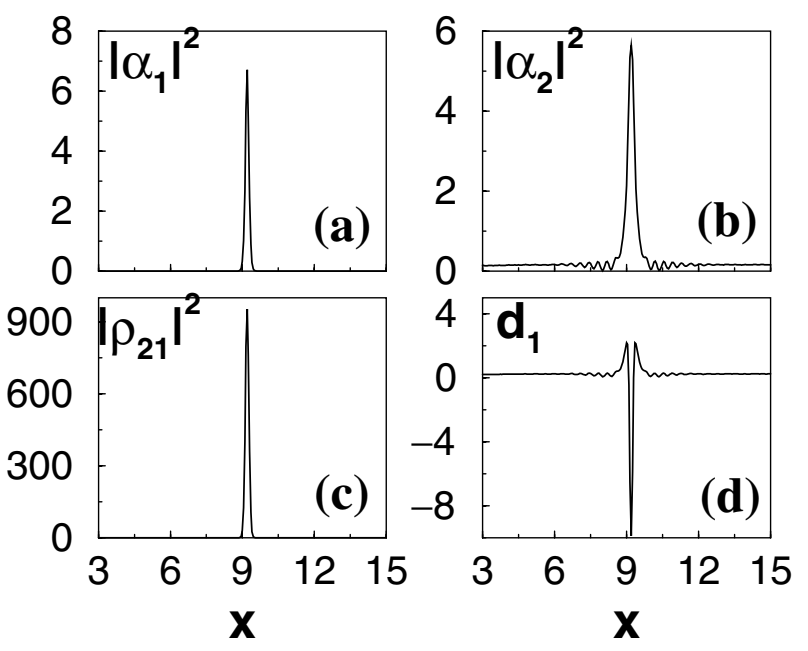

FIG. 5. Radial 1D section of the 2D CS of Fig. 4. 
(a)

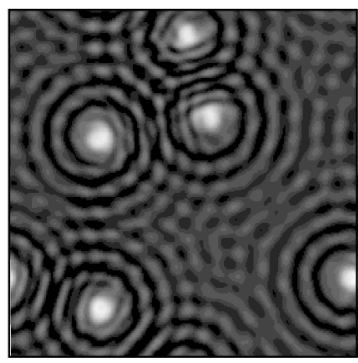

(b)

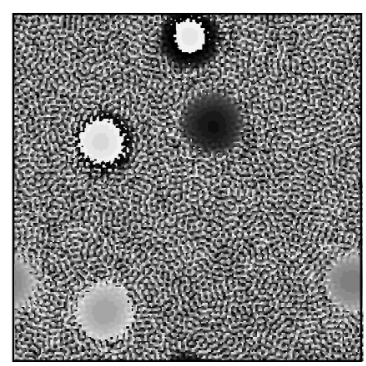

FIG. 6. (a) Intensity of field 2 and (b) phase of field 1 for the parameters of Fig. 4(a), except $r_{2}=184$.

a time-averaged maximum intensity of the field. A snapshot of this situation is shown in Fig. 6(a). The corresponding Fig. 6(b) shows that CSs do not lock their phases to a common value, in contrast with passive cavities [4]; this is probably due to the lack of a phase-reference background field.

For even larger pump rates ( $r_{2} \gtrsim 190$ in this case), CSs widen and start to lose their circular shape. For $r_{2} \gtrsim$ 200, most of the transverse plane becomes illuminated and the dynamics is dominated by optical vortices [16]. The CS state described here persists up to very large values $(\sim-50)$ of the cavity detuning $\delta_{2}$, provided $\delta_{1}$ remains relatively close to $\delta_{1}^{+}$(for other values of $\delta_{1}$, only uniform traveling-wave solutions are found [17]).

In conclusion, we have shown that cavity solitons can appear spontaneously in purely active optical media, as a result of the interplay between two-photon and singlephoton processes. The recent experimental realization of two-photon amplification [18] and lasing [19] sheds hope on the possibility of observing this phenomenology.

We acknowledge financial support from DGESIC (Spain) and Generalitat de Catalunya.

[1] G. I. Stegeman and M. Segev, Science 286, 1518 (1999).

[2] L. A. Lugiato, M. Brambilla, and A. Gatti, Adv. At. Mol. Opt. Phys. 40, 229 (1999); W. Firth and G. Harkness, Asian J. Phys. 7, 665 (1998).

[3] N. N. Rosanov and G. V. Khodova, J. Opt. Soc. Am. B 7, 1065 (1990); M. Tlidi, P. Mandel, and R. Lefever, Phys. Rev. Lett. 73, 640 (1994); M. Kreuzer, H. Gottschling,

T. Tschudi, and R. Neubecker, Mol. Cryst. Liq. Cryst. 207, 219 (1991); W. J. Firth and A. J. Scroggie, Phys. Rev. Lett. 76, 1623 (1996); M. Brambilla, L. A. Lugiato, and M. Stefani, Europhys. Lett. 34, 109 (1996).

[4] M. Brambilla, L. A. Lugiato, F. Prati, L. Spinelli, and W. J. Firth, Phys. Rev. Lett. 79, 2042 (1997); D. Michaelis, U. Peschel, and F. Lederer, Phys. Rev. A 56, R3366 (1997).

[5] A. Schreiber, B. Thuring, M. Kreuzer, and T. Tschudi, Opt. Commun. 136, 415 (1997); B. Schäpers, M. Feldmann, T. Ackemann, and W. Lange, Phys. Rev. Lett. 85, 748 (2000); R. Gallego, M. San Miguel, and R. Toral, Phys. Rev. E 61, 2241 (2000); V. J. Sánchez-Morcillo, I. PérezArjona, F. Silva, G. J. de Valcárcel, and E. Roldán, Opt. Lett. 25, 957 (2000).

[6] M. Saffman, D. Montgomery, and D.Z. Anderson, Opt. Lett. 19, 518 (1994).

[7] V. J. Sánchez-Morcillo, E. Roldán, G. J. de Valcárcel, and K. Staliunas, Phys. Rev. A 56, 3237 (1997); S. Longhi, Phys. Scr. 56, 611 (1997); G. L. Oppo, A. J. Scroggie, and W. J. Firth, J. Opt. B 1, 133 (1999); M. Le Berre, D. Leduc, E. Ressayre, and A. Tallet, J. Opt. B 1, 153 (1999); C. Etrich, U. Peschel, and F. Lederer, Phys. Rev. Lett. 79, 2454 (1997).

[8] V. B. Taranenko, K. Staliunas, and C. O. Weiss, Phys. Rev. Lett. 81, 2236 (1998).

[9] V. Y. Bazhenov, V. B. Taranenko, and M. V. Vasnetsov, Proc. SPIE 1840, 183 (1992); N. N. Rosanov, S. V. Fedorov, and G. V. Khodova, Physica (Amsterdam) 96D, 272 (1996); V. B. Taranenko, K. Staliunas, and C. O. Weiss, Phys. Rev. A 56, 1582 (1997).

[10] P. K. Jakobsen, J. V. Moloney, A. C. Newell, and R. Indik, Phys. Rev. A 45, 8129 (1992).

[11] J. García-Ojalvo, M. C. Torrent, R. Vilaseca, and M. Brambilla, Chaos Solitons Fractals 10, 819 (1999).

[12] J. García-Ojalvo, R. Vilaseca, and M. C. Torrent, Phys. Rev. A 56, 5111 (1997).

[13] J. García-Ojalvo, R. Vilaseca, and M. C. Torrent, Europhys. Lett. 43, 261 (1998).

[14] E. Roldán, G. J. de Valcárcel, R. Vilaseca, and V. Espinosa, J. Mod. Opt. 44, 83 (1997).

[15] M. Tlidi and P. Mandel, Phys. Rev. A 59, 2575 (1999).

[16] C. O. Weiss, M. Vaupel, K. Staliunas, G. Slekys, and V. B. Taranenko, Appl. Phys. B 68, 151 (1999).

[17] M. C. Torrent, R. Vilaseca, and J. García-Ojalvo, J. Opt. B 2, 432 (2000).

[18] O. Pfister, W. J. Brown, M. D. Stenner, and D. J. Gauthier, Phys. Rev. A 60, R4249 (1999).

[19] O. Pfister, W. J. Brown, M. D. Stenner, and D. J. Gauthier, Phys. Rev. Lett. 86, 4512 (2001). 